\title{
It Takes a Village: A Collaborative Approach to the COVID-19 Pandemic
}

\author{
Darshana T. Shah, PhD'
}

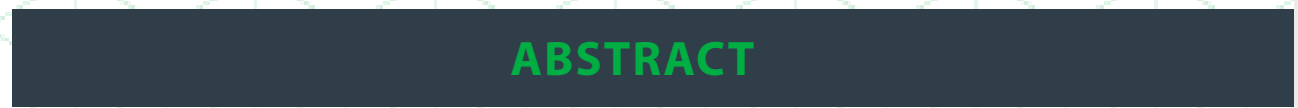

The COVID-19 pandemic has affected societies and health systems around the globe and resulted in a scenario of unprecedented interconnectedness among people, businesses, and ecosystems.

\section{KEYWORDS}

Teamwork, collaboration
Author affiliations are listed at the end of this article.

Corresponding Author: Darshana T. Shah, PhD Marshall University Joan C. Edwards School of Medicine shah@marshall.edu
The COVID-19 pandemic has affected societies and health systems around the globe and resulted in a scenario of unprecedented interconnectedness among people, businesses, and ecosystems. The pandemic has made it shockingly apparent how the intertwined and interdependent relationships that exist in health care between persons, businesses, academia, and government buffer the system from rapid and drastic change ${ }^{1}$. However, when we look to the future of health care globally in light of recent events, it is obvious that applying a team science approach to the pre-existing relationships in the healthcare system is key to solving the challenges facing society today.

The National Institute for Health defines team science ${ }^{2}$ as "collaborative efforts to address a scientific challenge that leverages the strengths and expertise of professionals trained in different fields." Team science offers tools for team building and collaboration across disciplinary, organizational, and cultural boundaries.

The COVID-19 pandemic has challenged all aspects of the healthcare system, from scientists tracking the virus' progress and variation, to medical students going through residency programs learning how to deliver effective patient care, to pharmacists tasked with delivering vaccines equitably. This issue of the Marshall Journal of Medicine highlights how these Covid-19 pandemic challenges have been addressed at multiple levels, and the authors detail how collaboration has been essential to their resolution.

Dr. Denver's editorial, "Leveraging a Public-Private-Academic Collaborative Partnership to Confront Challenges in the COVID-19 Pandemic," highlights the importance of collaboration in the quest to identify new virus variants in the state of West Virginia. Drs. Crump, Nims, and Hatler reflect on how a residency program realized the need to embrace telemedicine in the midst of the pandemic and detail the process and results of that effort in "Mr. Watson, come here - I want to see you": One rural residency program's rapid pivot to telemedicine during the pandemic." Dr. Massey gives us a perspective from the pharmacy side of medicine and muses on West Virginia's vaccination effort in his article, "Boots on the Ground and Shots in Arms: A West Virginia Pharmacist on COVID-19 Vaccinations." Finally, Dr. Hammad, et al. bring us perspective on adhering to and progressing surgical techniques in light of COVID-19 restrictions in 
their article, "Can the conventional cytology technique be sufficient in a center lacking ROSE?: Retrospective study during the COVID-19 pandemic." The varied perspectives represented in this issue of the Marshall Journal of Medicine remind us of the many facets of health care that work in tandem to progress science globally. When we realize that we share the common goals of delivering the best patient care possible and advancing processes and techniques in order to advance medical care, we can understand the importance of leaning into the relationships and collaborative opportunities available to us while embracing a team science approach to medicine.

\section{AUTHOR AFFILIATIONS}

1. Professor of Pathology, Associate Dean for Faculty, Marshall University Joan C. Edwards School of Medicine, Huntington, West Virginia

\section{REFERENCES}

1. Valeras AS. COVID-19: Complexity and the Black Swan. Fam Syst Health. 2020 Jun;38(2):221-223. doi: 10.1037/fsh0000486. PMID: 32525362.

2. Team Science - Office of Intramural Training \& Education at the National Institutes of Health (nih. gov) 